\title{
Tablas de mortalidad de Ecuador continental mediante un análisis de supervivencia
}

\section{Mortality tables of continental Ecuador using a survival analysis}

\author{
Francisco Vera \\ Mario Solórzano \\ Gina Ochoa \\ Sandra García Bustos \\ Solange Cevallos
}

\author{
Centro de Estudios e Investigaciones Estadísticas de la Escuela \\ Superior Politécnica del Litoral, Guayaquil, Ecuador
}

\section{Resumen}

Según investigaciones anteriores realizadas sobre mortalidad en Ecuador, existen tablas de mortalidad elaboradas de los datos del censo de 2001; sin embargo, no hay tablas actualizadas. Aunque las tablas de mortalidad son usualmente analizadas por sexo, existen otros factores que son influyentes. El objetivo de esta investigación es identificar si los factores tales como el sexo y el área de residencia influyen en la supervivencia de la población continental del Ecuador para 2010. A fin de determinar los factores que son influyentes en la supervivencia de la población fue utilizada la técnica de regresión logística y para la construcción de las tablas de mortalidad se utilizó el método clásico actuarial. El análisis muestra que el sexo, el área de residencia y su interacción, afectan la supervivencia de la población.

Palabras clave: Esperanza de vida, tablas de vida, regresión logística, método actuarial, supervivencia, método de Swanson.

\section{Abstract}

According to previous research carried out on mortality in Ecuador, the country has life tables drawn from the 2001 census data, however, there are no updated tables. Although mortality tables are usually analyzed by sex, there are other factors that are influential. This research goal is to identify whether factors such as sex and the area of residence influence the survival of the population of mainland Ecuador by 2010.To determine the factors that influence the survival of the population, the Logistic Regression technique was used, and for the construction of the mortality tables, the classic actuarial method is used. The analysis shows that sex, area of residence and its interaction, affect the survival of the population.

Key words: Life expectancy, life tables, logistic regression, actuarial method, survival-Swanson method. 


\section{INTRODUCCIÓN}

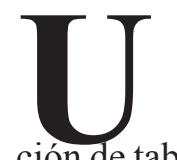

na de las principales variables consideradas al realizar un estudio demográfico en una población es la mortalidad. El estudio de esta variable se efectúa generalmente mediante la construcción de tablas cuyos indicadores expresan el riesgo de fallecer o la esperanza de vida de una población definida, registrada por edad o grupos de edad. De aquí que, las tablas de mortalidad son de gran utilidad para estudios de población en el ámbito social y económico, así como también en el ámbito de los seguros como base para el cálculo de primas de los asegurados.

Según el Instituto Nacional de Estadística y Censos (INEC, 2006), en Ecuador disminuyó en 18 por ciento la mortalidad general en el periodo de 1990 a 2001, además la esperanza de vida al nacer aumentó de 68.9 a 73.8 años en el mismo periodo. Adicionalmente, estimaciones realizadas por la Comisión Económica para América Latina y el Caribe (CEPAL, 2004) indican que la esperanza de vida al nacer para Ecuador en el periodo 20052010 era de 75 años y en el periodo 2010-2015 de 75.8 años.

Este artículo utiliza datos registrados por el Instituto Nacional de Estadística y Censos (INEC) correspondientes a estadísticas de nacimientos y defunciones, así como del censo de población. En la primera parte del estudio se realiza una revisión bibliográfica de estudios realizados en Ecuador respecto a la construcción de tablas de mortalidad e investigaciones desarrolladas en otros países respecto al análisis de supervivencia. En segundo lugar, la técnica de Regresión Logística es utilizada para analizar si factores como el sexo o el área de residencia en el que vive la población ecuatoriana influye en la probabilidad de supervivencia; y, a partir del resultado obtenido en este análisis, se construyen tablas de mortalidad y se calcula la esperanza de vida al nacer para el Ecuador para la población del Ecuador a través del método clásico actuarial y del método de Swanson.

\section{Antecedentes}

En Ecuador, uno de los estudios realizados respecto a tablas de mortalidad revela que para el año 1990 la esperanza de vida al nacer de un ecuatoriano era de 72 años, y según las estimaciones de la probabilidad de fallecimiento alrededor de dos por ciento de los recién nacidos se esperaba muriesen antes de cumplir el primer año de vida; por otro lado, el máximo número 
de muertes ocurrían aproximadamente en la edad de 88 años tanto para la población general como para la población de hombres, existiendo una diferencia de un año con respecto a las mujeres, puesto que el máximo número de muertes estaba en la edad de 87 años (Sánchez, 2000: 77).

Capa y Lara (2004) elaboraron tablas de mortalidad para el país en base a los datos del censo 2001, así como estadísticas del número de nacidos vivos y de las defunciones para los años 2000, 2001 y 2002, además compararon las esperanzas de vida al nacer para la población total y la población por sexo y área geográfica.

Siguiendo con el análisis de las tablas de mortalidad en el tiempo, otro de los estudios desarrollados en este ámbito muestra que, en el año 2001, la esperanza de vida al nacer para la población total del Ecuador era de 73.7 años; además, comparando entre hombres y mujeres, se tiene una brecha de aproximadamente seis años en relación con la esperanza de vida, prevaleciendo las mujeres sobre los hombres. En este mismo estudio, se construyeron tablas de mortalidad para los años 2005, 2010, 2015 y 2020 , en ellas se evidencia el crecimiento de la esperanza de vida de 74.5 años en el 2005 a 76.9 años en 2020 (Rivadeneira, 2008: 20).

Por otra parte, existen estudios para otros países en los que se analizan otros factores distintos del género, por ejemplo considerando un país altamente desarrollado como es Estados Unidos, un estudio realizado por Singh y Siahpush (2014) presenta las tendencias en las disparidades de la esperanza de vida al nacer en este país entre las áreas urbana y rural, llegando a la conclusión que la esperanza de vida entre los años 2005 y 2009 es mayor para las personas que residen en grandes áreas metropolitanas, siendo igual a 79.1 años, en comparación con los 76.9 años que resultan en las ciudades pequeñas urbanas y 76.7 años en las zonas rurales.

Algunas investigaciones donde se estudian los factores que inciden en la mortalidad de una población utilizan la técnica de Regresión Logística: Guilkey y Rindfuss (1987) analizaron el uso de esta técnica para estimar un modelo de riesgo discreto. En otra de ellas se usa esta técnica para estimar la mortalidad mediante variables demográficas como género, edad y provincia de procedencia. En este estudio se desarrolló una metodología para reducir el sesgo debido a casos con valores desconocidos de factores demográficos en el registro de la defunción (Chutinantakul et al., 2014: 6).

Zhu y Zhi (2014) analizaron la mortalidad de la población asegurada de Estados Unidos utilizando un modelo de regresión logística y compararon los resultados del modelo obtenido aplicado en datos de industria con los valores de tabla de mortalidad estándar establecidos por la Sociedad de 
Actuarios. Este estudio concluyó que si se diseña un modelo apropiado mediante esta técnica se obtienen muy buenos resultados. Cabe mencionar que la población asegurada en Estados Unidos se estimó para 2016 en 91.6 por ciento de los estadounidenses siendo la única nación industrializada en el mundo sin cobertura universal de atención médica (Bureau, US Census, 2016).

En el año 2015 la revista Nutrición Hospitalaria publicó un artículo acerca de los factores que influyen en la mortalidad perinatal en gestantes de Colombia. En la investigación se asociaron factores como el sexo del recién nacido, la región geográfica, el nivel socioeconómico de la madre, el seguimiento prenatal, entre otros, llegándose a la conclusión general que las variables sociodemográficas influyen en la mortalidad (Navarro et al., 2015: 8). Por último, Acheampong y Avorgbedor (2017) hicieron un análisis retrospectivo de la mortalidad en menores de cinco años en Ghana usando la Encuesta Demográfica y de Salud (1988-2014). En ese trabajo se determinó que factores como la edad materna, la duración de lactancia y el tipo de parto incidieron en la mortalidad.

Entonces, resulta pertinente la aplicación de la técnica de Regresión Logística para determinar si otros factores distintos al género pueden influir en la mortalidad del Ecuador. En el presente trabajo se analizan otros factores como el área y región de residencia, por tanto, esta investigación es relevante para el sistema de salud pública, aseguradoras públicas y privadas, y en el desarrollo económico y social del Ecuador.

\section{Datos}

El estudio está basado en los datos del Censo de Población y Vivienda (CPV) realizado el 28 de noviembre de 2010 por el Instituto Nacional de Estadística y Censos, ente responsable de llevar las estadísticas del Ecuador; además, se utilizan los datos de las estadísticas vitales de nacimientos y defunciones correspondientes al mes de diciembre de 2010 y a los meses de enero a noviembre de 2011. Los datos de los nacimientos son utilizados únicamente al construir las tablas de mortalidad, mientras que las bases de datos del censo de población y las defunciones se utilizan tanto en la construcción de tablas de mortalidad como en la identificación de los factores que influyen en la supervivencia. 


\section{MÉtodos}

\section{Factores que inciden en la supervivencia}

Con el fin de determinar los factores que inciden en la supervivencia de la población del Ecuador se utiliza la técnica Regresión Logística, modelo predictivo que se utiliza cuando la variable de respuesta es binaria pues sólo toma dos valores, 0 si no presenta la característica de interés o 1 si la presenta; es decir, a través de este modelo se intenta modelar la probabilidad de conseguir un determinado resultado, por ejemplo: vive o muere, gana o pierde, tiene o no una enfermedad, etc. (Wilson y Lorenz, 2015).

El modelo de Regresión Logística puede ser definido en base a la función logística, función que describe la forma matemática del modelo, y cuyo dominio son los números reales y su rango se encuentra en el intervalo $(0,1)$. La expresión matemática del modelo logístico es la siguiente:

$$
p(\boldsymbol{X})=1 /\left(1+e^{-\left(\beta_{0}+\sum_{i=1}^{k} \beta_{i} X_{i}\right)}\right)
$$

donde $\mathrm{X}_{1}, \mathrm{X}_{2}, \ldots, \mathrm{X}_{\mathrm{k}}$ representan las variables independientes o de explicación; y $\beta_{1}, \beta_{2}, \ldots, \beta_{\mathrm{k}}$ son términos constantes que representan los parámetros desconocidos del modelo de regresión (Kleinbaum y Klein, 2010).

Una expresión general del modelo logístico puede obtenerse a través de la transformación logit, misma que está definida como logit $p$ y es igual a:

$$
\text { Logit } p(X)=\log _{e}(p(X) /(1-p(X))
$$

En un modelo de Regresión Logística se intenta siempre comparar dos individuos o grupos de individuos, para esto se define una expresión denominada razón de momios y en inglés odds ratio (OR), misma que no es más que la razón entre dos odds (razón de la probabilidad de que un evento ocurra sobre la probabilidad de que no ocurra) (Kleinbaum y Klein, 2010).

Dado un modelo logístico en su forma general $\mathrm{p}(\mathrm{X})$, la expresión matemática para calcular el odds ratio es:

$$
\mathrm{OR}=\left(\mathrm{p}\left(\mathrm{X}_{1}\right) /\left(1-\mathrm{p}\left(\mathrm{X}_{1}\right)\right)\right) /\left(\mathrm{p}\left(\mathrm{X}_{2}\right) /\left(1-\mathrm{p}\left(\mathrm{X}_{2}\right)\right)\right)
$$

\section{Construcción de tablas de mortalidad}

Uno de los objetivos planteados en esta investigación es construir tablas de mortalidad considerando ciertas características que pueden influir en la 
mortalidad como: la edad, el sexo y el área de residencia de los individuos de la población. Las tablas de mortalidad tienen como objetivo estimar el tiempo de vida promedio mediante el uso de tasas de supervivencia para una edad específica o para un rango de edad de una población dada (Yusuf, Martins y Swanson, 2014).

Una tabla de mortalidad de manera usual, contiene las siguientes funciones $\mathrm{q}_{\mathrm{x}}, \mathrm{p}_{\mathrm{x}}, \mathrm{d}_{\mathrm{x}}, 1_{\mathrm{x}}$, donde $q_{\mathrm{x}}$ representa la probabilidad que una persona de edad $x$ fallezca antes de cumplir la edad $x+1, p_{x}$, es la probabilidad que una persona sobreviva a la edad $x+1, d_{\mathrm{x}}$ el número de muertes entre las edades $x$ y $x+1$; y $l_{\mathrm{x}}$, el número de sobrevivientes a la edad $x$. Cada una de estas expresiones están ligadas con una función de probabilidad denominada función de supervivencia, la misma que se define como:

$$
\mathrm{s}(\mathrm{x})=1-\mathrm{F}(\mathrm{x})=\mathrm{P}(\mathrm{X}>\mathrm{x}), \mathrm{x} \geq 0
$$

Para construir las tablas de mortalidad por género y área, se utiliza el método actuarial clásico, el cual implica el análisis de diversos elementos que constituyen la tabla de vida; el primero de ellos y base para el cálculo de los indicadores restantes se denomina tasa de muerte específica por edad y se define como $\mathrm{m}_{\mathrm{x}}=\mathrm{d}_{\mathrm{x}} / \mathrm{P}_{\mathrm{x}}$, donde $P_{\mathrm{x}}$ es el número de personas en la población a la edad $x$.

Una vez obtenida la tasa de mortalidad, se procede a calcular la probabilidad de morir entre la edad $x$ y $x+1$, la misma que está definida por:

$$
q_{x}=d_{x} /\left(P_{x}+\frac{1}{2} d_{x}\right)
$$

Generalmente los valores de $q_{\mathrm{x}}$ presentan irregularidades, las que no corresponden a características reales de la población, sino más bien al procedimiento de interpolación y a errores de diverso tipo que presenta la información básica (Capa y Lara, 2004). Por ello es necesario suavizarla. En la investigación realizada, primero se calcularon los $\mathrm{q}_{\mathrm{x}}$ para un año antes $\mathrm{y}$ después del censo por género y área, y se calculó un promedio entre las tres probabilidades de muerte para suavizar la serie. Otras técnicas matemáticas de suavizamiento se pueden encontrar en (Silva et al., 2014; Guerrero y Silva, 2015; Debón et al., 2006)

Luego del suavizamiento de los valores de $q_{\mathrm{x}}$, se puede definir la probabilidad de supervivencia como $\mathrm{p}_{\mathrm{x}}=1-\mathrm{q}_{\mathrm{x}}$ (Yusuf, Martins y Swanson, 2014). 
Considerando una población inicial de 100 mil habitantes denotada por $l_{0}$ (número de sobrevivientes a la edad 0 ), se define el número de muertes que ocurren entre la edad $x$ y $x+1$ como $\mathrm{d}_{\mathrm{x}}=\mathrm{q}_{\mathrm{x}} \times 1_{\mathrm{x}}$. Con estos valores se calcula el número de personas-año vividos de los individuos que han sobrevivido al inicio de ese año y puede ser estimado como la suma entre el número de sobrevivientes a la edad $x+1$ más el periodo promedio de vida de aquellos habitantes que fallecen a la edad $x$.

A partir de los valores de las personas-año vividos más allá de la edad $x$ se facilita el cálculo del total de personas-año vividos $T_{x}$, siendo este igual $\mathrm{a} \mathrm{L}_{\mathrm{x}}+\mathrm{L}_{\mathrm{x}+1}+\ldots+\mathrm{L}_{\mathrm{w}}$, donde $w$ representa la edad máxima. Utilizando las definiciones de $T_{\mathrm{x}}$ y $L_{\mathrm{x}}$ y se define el número medio de personas-año vividos más allá de la edad $x$, conocido también como esperanza de vida, de la siguiente manera $e_{x}=T_{x} / L_{x}$ (Yusuf, Martins y Swanson, 2014).

\section{Método de Swanson}

Para determinar la esperanza de vida del Ecuador global y por sexo, se utilizó el método de Swanson, este método requiere los datos oficiales de las esperanzas de vida para su aplicación, situación que no hace posible la obtención de un modelo para estimarlas por área puesto que el ente oficial de estadísticas del país, Instituto Nacional de Estadística y Censos, no cuenta con los valores de esperanzas de vida segregados por esta variable.

El método de Swanson es una regresión no lineal para estimar la esperanza de vida al nacer con variables explicativas: la tasa bruta de mortalidad (CDR por sus siglas en inglés), definida como el total de defunciones entre el total de población (se utiliza población a mitad de año), y el porcentaje de la población con 65 o más años (P65+); ambas observadas en un mismo año calendario (Swanson, 1989; Paredes y Silva, 2017).

Los supuestos del modelo son los mismos de un modelo de regresión lineal clásico, errores normalmente distribuidos, homocedástico y no correlacionados (Davidian y Giltinan, 1995), siendo la ecuación del modelo:

$\mathrm{e}_{0}=\beta_{0}+\mathrm{b}_{1} \mathrm{CDR}+\beta_{2} \mathrm{CDR}^{2}+\beta_{3} \ln (\mathrm{P} 65+)+\beta_{4} \ln (\mathrm{P} 65+)^{2}+\beta_{5} \ln (\mathrm{P} 65+)^{2}$ $+\beta_{6} \ln (\mathrm{P} 65+) \mathrm{CDR}+\mathrm{e}$

El modelo no resulta apropiado si: a) la población está envejecida con más de 20 por ciento de población de 65 o más años; y b) la comunidad es pequeña y con pocas muertes. 


\section{Resultados}

\section{Análisis descriptivo}

El censo de población y vivienda de Ecuador realizado en el año 2010 revela que el número de habitantes del país en ese año, en las regiones Costa, Sierra y Oriente, era de $14 ' 401,899$, donde 50.2 por ciento se concentra en la Costa, 44.7 por ciento en la Sierra y 5.1 por ciento restante en el Oriente. En Ecuador continental la mayoría de la población es de género femenino (50.5 por ciento), sin embargo, la diferencia es mínima con respecto a los hombres puesto que representan 49.5 por ciento de la población. En relación con el área, 62.8 por ciento de los habitantes residen en el área urbana y apenas 37.2 por ciento en el área rural (Figura 1).

La Figura 2 muestra la proporción de habitantes por sexo y área de residencia en las tres regiones de Ecuador continental. La Sierra es la región con mayor proporción de mujeres y el Oriente la región de mayor proporción de hombres; la mayoría de los habitantes de las regiones Costa y Sierra vive en el área urbana, mientras que los habitantes del Oriente en su mayoría (61.1 por ciento) vive en el área rural.

Del total de habitantes del Ecuador continental, 62,066 fallecieron un año después del censo realizado en 2010 , lo que representa 0.43 por ciento de la población. En la Figura 3 se puede observar que las defunciones mantienen la misma distribución de datos que la población total, tanto para el sexo como el área de residencia; destacando que la proporción de hombres que fallecen es superior a las mujeres y que la proporción de habitantes que fallecen en el área urbana es superior a los que viven el área rural.

\section{Factores que inciden en la supervivencia}

Acorde a los objetivos de esta investigación, se plantean tres modelos de Regresión Logística para determinar si los factores sexo y área de residencia influyen en la supervivencia de la población. La variable a ser explicada es el estado de supervivencia de los habitantes, que toma el valor de uno si vive y cero si muere, y como variable de explicación se tiene la edad segmentada en tres y cinco grupos elegidos a partir del análisis visual de los patrones que sigue la supervivencia de la población del Ecuador continental. 
Tablas de mortalidad de Ecuador continental mediante un análisis de supervivencia ... / F. VERA et al.

Figura 1: Proporción de habitantes por región, sexo y área de residencia
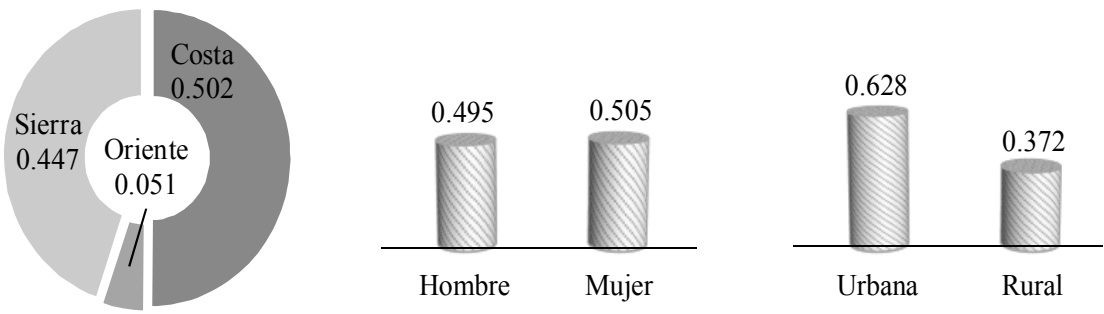

Fuente: Elaboración del autor a partir de los datos del CPV 2010.

Figura 2: Proporción de habitantes por sexo y área de residencia

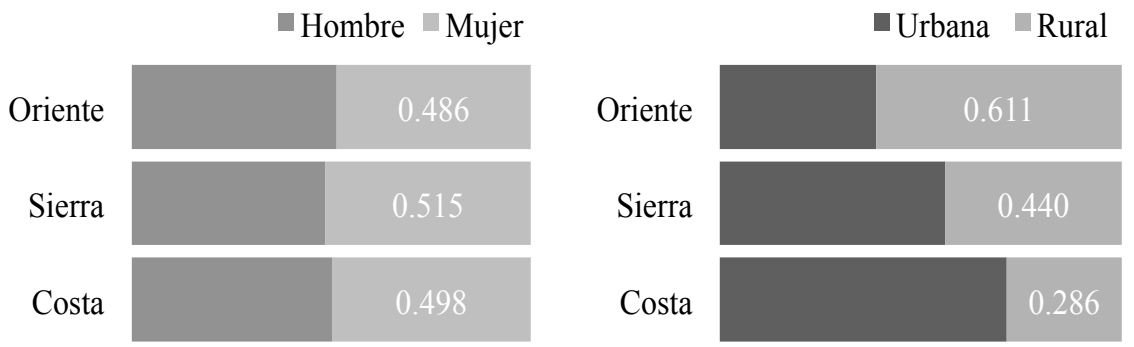

Fuente: elaboración del autor a partir de los datos del CPV 2010.

Figura 3: Proporción de defunciones por sexo y área de residencia

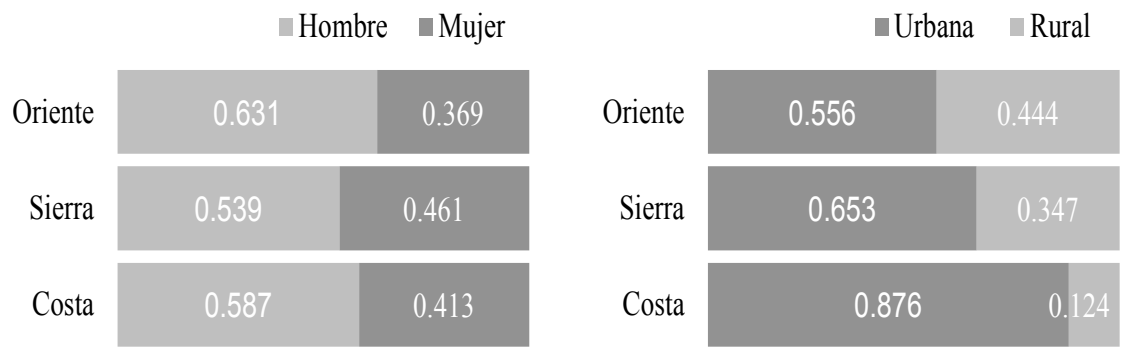

Fuente: elaboración del autor a partir de los datos de las Defunciones Generales 2010 y 2011. 
La segmentación en tres grupos corresponde si la persona tiene 0 años, 1 a 16 años o más de 17 años, considerándose un primer caso donde se plantea un modelo lineal simple para los dos primeros intervalos y para el tercero un modelo cuadrático; y un segundo caso con un modelo lineal simple para la edad 0 años y un modelo cuadrático para los intervalos de edad 1 a 16 años o más de 17 años. Para la segmentación de cinco grupos los rangos de edad son 0 años, 1 a 9 años, 10 a 16 años, 17 a 50 años o más de 51 años, considerándose un modelo lineal simple en cada uno.

Las estimaciones $\hat{\beta}_{i}$ de los parámetros de Regresión Logística son obtenidas por el método de máxima verosimilitud, por tanto, tienen distribución asintóticamente normal (Newey y McFadden, 1994). Para probar la significancia de las variables explicativas (contraste de hipótesis: $\mathrm{H}_{0}: \mathrm{b} i=0$ y $\mathrm{H}_{1}: \mathrm{b} i \neq 0$ ) se usó el estadístico:

$$
Z=\frac{\hat{\beta}_{i}}{\sigma_{\beta_{i}}}
$$

Analizando la devianza, el AIC (criterio de información akaike) y el BIC (criterio de información bayesiano) de cada modelo se establece uno que sea la base para explicar el estado de supervivencia en términos de la edad, notándose claramente en la Tabla 1 que el modelo con dos componentes cuadráticos es el mejor, ya que presenta la menor devianza, el menor AIC y BIC (Kleinbaum y Klein, 2010; Vrieze, 2012).

Tabla 1: Comparación de modelos estado de supervivencia versus edad

\begin{tabular}{lrrr}
\hline Modelo & Devianza & AIC & BIC \\
\hline Con dos componentes lineales & 25016.65 & 46490.37 & 46531.66 \\
Con dos componentes cuadráticos & 24454.84 & 45930.56 & 45978.73 \\
Con cinco componentes lineales & 25357.98 & 46837.70 & 46899.63 \\
\hline
\end{tabular}

Fuente: elaboración del autor a partir de los datos del CPV 2010 y Defunciones Generales 2010 y 2011.

A partir del modelo base seleccionado, se adicionan las variables sexo y área de residencia para determinar si inciden en la supervivencia de la población. Para el caso del sexo, la categoría de referencia es ser hombre y para la variable área de residencia es vivir en el área urbana. De acuerdo con los resultados del modelo de regresión, para la población total del Ecuador continental, una mujer es 1.49 veces más propensa a sobrevivir que un hombre, y una persona que vive en el área rural es 2.13 veces más propensa a sobrevivir que una persona que viva en el área urbana. La propensión a sobrevivir para una mujer vista por regiones es mayor en 
la Costa y Oriente, puesto que presentan un odd ratio igual a 1.54 y 1.59 respectivamente; $\mathrm{y}$, respecto al área, la región Costa presenta una marcada diferencia con respecto a las otras regiones (Tabla 2).

Tabla 2: Odds Ratio de los modelos logísticos para sexo y área de residencia

\begin{tabular}{|c|c|c|c|}
\hline Sexo & Odds Ratio & Área de residencia & Odds Ratio \\
\hline País & & País & \\
\hline Ecuador & 1.49 & Ecuador & 2.13 \\
\hline Región & & Región & \\
\hline Costa & 1.54 & Costa & 3.15 \\
\hline Sierra & 1.43 & Sierra & 1.70 \\
\hline Oriente & 1.59 & Oriente & 1.94 \\
\hline
\end{tabular}

Los modelos individuales de sexo y área muestran que ambas variables influyen en la supervivencia de la población del país; a continuación, se analizan de manera conjunta y para ello se plantea un modelo aditivo y otro con interacción. Ambos modelos resultan significativos, es decir, hay un efecto del sexo para los que residen en el área urbana y otro para los del área rural.

Los odds ratio para el modelo de interacción muestran que un hombre que vive en el área rural es 2.3 veces más propenso a sobrevivir que un hombre que vive en el área urbana, y en el caso de las mujeres, una que vive en el área rural es casi dos veces más propensa a sobrevivir que una que vive en el área urbana. Respecto al efecto del sexo, una persona que vive en el área urbana y es mujer es 1.58 veces más propensa a sobrevivir que una persona que vive en el área urbana y es hombre; $y$, una persona que vive en el área rural y es mujer es 1.39 veces más propensa a sobrevivir que una persona que vive en el área rural y es hombre.

De acuerdo a la información previa, la probabilidad de supervivencia es mayor para los habitantes que viven en el área rural; sin embargo, la diferencia es superior en los hombres; desde el punto de vista del sexo, la probabilidad de supervivencia es mayor en las mujeres, pero la diferencia es mayor para los que viven en el área urbana (Tabla 3).

El modelo que presentó un mejor ajuste es:

$$
P(Y=1 \mid \text { Edad,Sexo, Area })=\frac{1}{1+e^{-(A)}}
$$


Donde:

$$
\begin{aligned}
A= & I_{0} \beta_{0}+I_{1-16}\left(\beta_{1}+\beta_{2} *(\operatorname{Edad}-10)+\beta_{3} *(\operatorname{Edad}-10)^{2}\right) \\
& +I_{\geq 17}\left(\beta_{4}+\beta_{5} *(\operatorname{Edad}-40)+\beta_{6} *(\operatorname{Edad}-40)^{2}\right)+\beta_{7} * \text { Sexo }+\beta_{8} \\
& * \text { Área }+b_{9} * \text { Sexo }+ \text { Área }
\end{aligned}
$$

Las estimaciones de los coeficientes del modelo y el valor $p$ de las pruebas $Z$ se presentan en la Tabla 4 . Se puede notar que todas las variables resultan significativas. La devianza para este modelo es 14,864 y un valor de AIC de 36,346.

Tabla 3: Odds Ratio del modelo logístico con interacción sexo y área

\begin{tabular}{lllll}
\hline & País & \multicolumn{3}{l}{ Regiones } \\
\hline Odds Ratio & Ecuador & Costa & Sierra & Oriente \\
\hline Efecto Área (Hombre) & 2.30 & 3.45 & 1.75 & No significativo \\
Efecto Área (Mujer) & 2.01 & 2.99 & 1.67 & \\
Efecto Sexo (Urbana) & 1.58 & 1.66 & 1.47 & \\
Efecto Sexo (Rural) & 1.39 & 1.44 & 1.40 & \\
\hline
\end{tabular}

Nota: categoría de referencia sexo = hombre, área = urbana.

Fuente: elaboración del autor a partir de los datos del CPV 2010 y Defunciones Generales 2010 y 2011.

Tabla 4: Coeficientes del mejor modelo ajustado

\begin{tabular}{lrrrr}
\hline Coeficientes & $\begin{array}{c}\text { Estimador } \\
\text { de beta }(\hat{\boldsymbol{\beta}})\end{array}$ & $\begin{array}{r}\text { Error } \\
\text { Estándar }(\hat{\boldsymbol{\beta}})\end{array}$ & $\begin{array}{r}\text { Estadístico } \\
\text { de Prueba }\end{array}$ & Valor p \\
\hline Intercepto & 3.978 & 0.019 & 211.681 & 0.000 \\
$\mathrm{I}_{1-16}$ & 3.718 & 0.039 & 96.107 & 0.000 \\
$\mathrm{I}_{1-16} *($ Edad -10$)$ & -0.033 & 0.005 & -6.619 & 0.000 \\
$\mathrm{I}_{1-16} *(\text { Edad }-10)^{2}$ & -0.023 & 0.001 & -23.813 & 0.000 \\
$\mathrm{I}_{217}$ & 1.701 & 0.020 & 87.182 & 0.000 \\
$\mathrm{I}_{117} *($ Edad -40$)$ & -0.043 & 0.000 & -104.137 & 0.000 \\
$\mathrm{I}_{217} *(\text { Edad }-40)^{2}$ & -0.001 & 0.000 & -91.052 & 0.000 \\
Sexo $=$ mujer & 0.460 & 0.010 & 47.940 & 0.000 \\
Área = rural & 0.831 & 0.013 & 64.917 & 0.000 \\
Sexo*Área & -0.131 & 0.019 & -6.761 & 0.000 \\
\hline
\end{tabular}

Nota: categoría de referencia sexo = hombre, área = urbana.

Fuente: elaboración del autor a partir de los datos del CPV 2010 y Defunciones Generales 2010 y 2011. 


\section{Tablas de mortalidad}

Con el objetivo de analizar la probabilidad de morir y la esperanza de vida en las poblaciones correspondientes a las variables que resultaron de influencia en la supervivencia de los habitantes de Ecuador continental, se construyen tablas de mortalidad considerando como población expuesta al riesgo de morir al inicio del año el número de habitantes empadronados en el censo de 2010, y como población de muertes los datos registrados en las estadísticas vitales de defunciones del año 2010 y 2011 publicadas por el Instituto Nacional de Estadística y Censos (INEC). En la Figura 4 se presenta la probabilidad de fallecimiento con un filtro de cinco términos para las variables sexo y área, donde el eje de las abscisas representa la edad y el eje de las ordenadas el logaritmo de la probabilidad de muerte; nótese que en la mayoría de las edades los hombres y los habitantes que viven en el área urbana son los que tienen mayores probabilidades de morir.

Figura 4: Probabilidad de fallecer por sexo y área
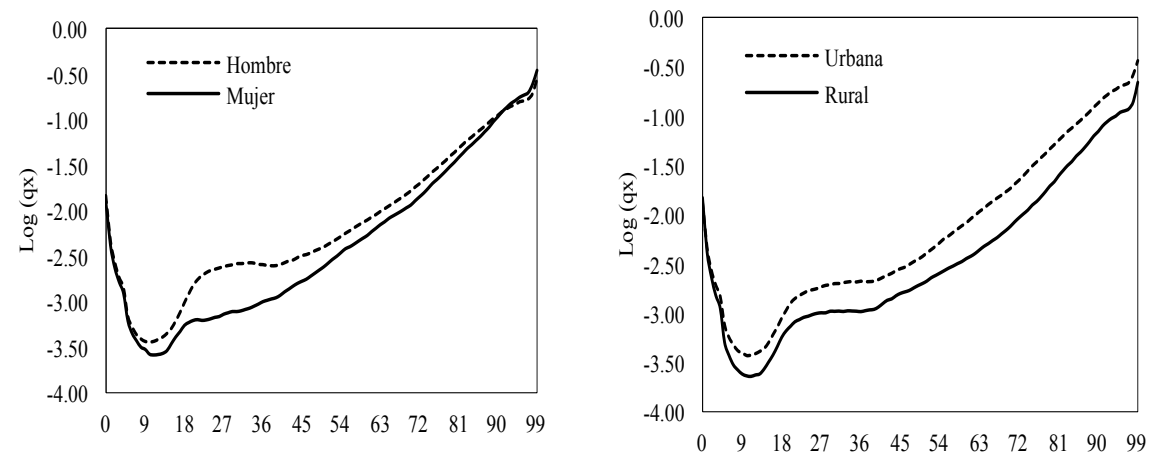

Fuente: elaboración del autor a partir de los datos del CPV 2010 y Defunciones Generales 2010 y 2011.

En cuanto a la esperanza de vida se obtienen resultados similares puesto que los hombres y los habitantes del área urbana presentan valores por debajo de las mujeres y los habitantes del área rural. Se presenta en la Figura 5 la esperanza de vida para las variables sexo y área de residencia, de la misma manera que en la figura de la probabilidad de fallecimiento, se representa en el eje de las $X$ la edad de los habitantes del Ecuador continental y en el eje de las $Y$ los valores de la esperanza de vida, destacando que la esperanza de vida al nacer del país es 77.9 años. 
Figura 5: Esperanza de vida por sexo y área
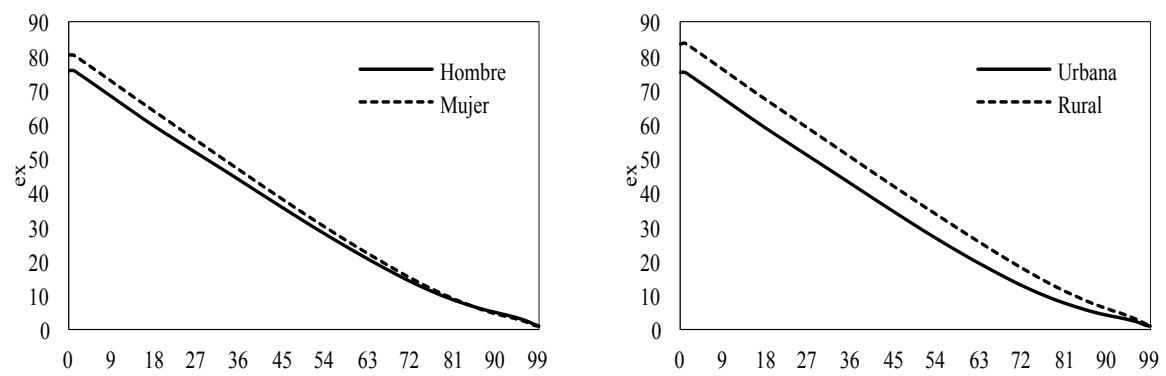

Fuente: elaboración del autor a partir de los datos del CPV 2010 y Defunciones Generales 2010 y 2011.

Figura 6: Probabilidad de Fallecer y Esperanza de Vida (Interacción sexo-área)
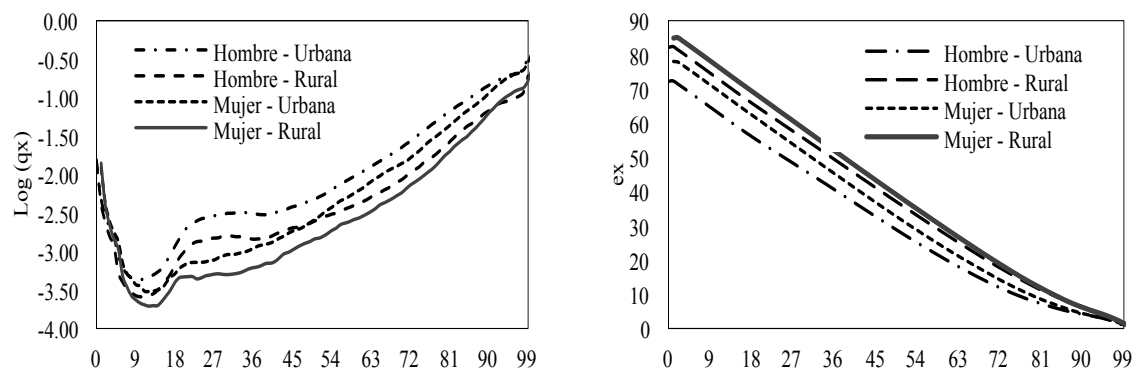

Fuente: elaboración del autor a partir de los datos del CPV 2010 y Defunciones Generales 2010 y 2011.

El efecto de la interacción sexo-área de residencia en términos de la probabilidad de fallecimiento y la esperanza de vida se observa en la Figura 6 , donde se nota que un hombre que vive en el área urbana tiene la mayor probabilidad de fallecimiento y una mujer que vive en el área rural tiene la menor probabilidad de fallecer; estos valores se pueden corroborar observando la Figura 6.

Por otra parte, el método de Swanson fue utilizado para obtener una estimación de la esperanza de vida al nacer para hombres, mujeres y para todo Ecuador. Como se mencionó antes no se realizó esta metodología por áreas debido a que no se encuentra un registro oficial de esperanza de vida al nacer para ellas. Los datos oficiales de Esperanza de vida al nacer para 2010 fueron tomados del compendio estadístico del INEC 2015 (Instituto Nacional de Estadística y Censos, 2015). 
Los modelos construidos cumplen con los supuestos de normalidad, homocedasticidad y no correlación de los residuos. Sin embargo, presentan problemas de multicolinealidad, es decir, alta correlación entre variables explicativas. Cabe mencionar que la multicolinealidad afecta al cálculo de la estimación de varianza de los estimadores y a la estimación de los efectos de las variables explicativas colineales sobre la esperanza de vida al nacer, por tanto, afecta a las pruebas de significancia de las variables (Gujarati and Porter, 1991). Pero como el objetivo es sólo hacer una estimación puntual de la Esperanza de vida al nacer, los resultados no resultan afectados por la multicolinealidad.

Para la modelación se cumplieron todas las condiciones para las cuales esta metodología presenta resultados favorables y que fueron mencionadas anteriormente. Además, los valores de tasa bruta de mortalidad usados en la modelación fueron promediados previamente con los valores de tasa bruta de un año antes y después (Instituto Nacional de Estadística y Censos, 2012). El modelo de Swanson para todo Ecuador obtuvo un $\mathrm{R}^{2}$ de 69.33 por ciento, mientras que para el de mujeres fue de 70.47 por ciento y para el de hombres, de 74.77 por ciento (Tabla 5).

Se puede notar el buen ajuste para cada uno de los valores oficiales mediante esta técnica.

\section{Conclusiones}

El análisis descriptivo de la población de Ecuador continental revela que 0.43 por ciento $(62,066)$ falleció un año después del censo realizado en 2010. Tomando en cuenta los valores por edad tanto para las defunciones como la población censada, se encontró a través del modelamiento logístico que los factores sexo y área de residencia influyen en la supervivencia de la población del país de manera individual y en su interacción; además, la interacción entre estos dos factores es significativa en las regiones Costa y Sierra, no así para la región Oriente, donde la significancia sólo se presenta de manera individual.

Un odd ratio de 1.49 demuestra que una mujer tiene más alta posibilidad de sobrevivir que un hombre, y con el análisis referente al área de residencia encontramos que una persona que vive en el área rural es 2.13 veces más propensa a sobrevivir que una persona que viva en el área urbana. 
Tabla 5: Esperanza de vida al nacer oficiales y ajustadas con el Método de Swanson

\begin{tabular}{lcccccc}
\hline Provincia & \multicolumn{2}{c}{ Mujeres } & \multicolumn{2}{c}{ Hombres } & \multicolumn{2}{c}{ Nacional } \\
\hline & Oficial & Predicho & Oficial & Predicho & Oficial & Predicho \\
\hline Azuay & 80.2 & 79.2 & 74.2 & 73.4 & 77.2 & 76.4 \\
Bolivar & 76.6 & 76.6 & 72.7 & 72.6 & 74.6 & 74.3 \\
Cañar & 79.0 & 79.5 & 72.1 & 72.8 & 75.6 & 76.3 \\
Carchi & 77.0 & 78.5 & 74.2 & 75.5 & 75.6 & 77.1 \\
Cotopaxi & 76.7 & 77.4 & 70.4 & 71.5 & 73.5 & 74.5 \\
Chimborazo & 76.1 & 76.2 & 70.0 & 70.3 & 73.1 & 73.4 \\
El Oro & 77.6 & 77.9 & 72.0 & 72.1 & 74.8 & 74.9 \\
Esmeraldas & 76.2 & 76.3 & 69.1 & 70.6 & 72.7 & 73.5 \\
Guayas & 76.9 & 76.8 & 70.2 & 70.3 & 73.6 & 73.5 \\
Imbabura & 77.5 & 77.1 & 72.2 & 71.5 & 74.9 & 74.3 \\
Loja & 79.4 & 78.9 & 76.1 & 75.3 & 77.7 & 76.9 \\
Los Ríos & 75.0 & 75.8 & 68.6 & 67.9 & 71.8 & 72.0 \\
Manabi & 77.8 & 77.8 & 71.6 & 71.3 & 74.7 & 74.6 \\
Morona & 76.0 & 76.4 & 72.3 & 72.7 & 74.1 & 74.5 \\
Napo & 76.0 & 75.6 & 72.3 & 71.7 & 74.1 & 73.6 \\
Pastaza & 76.0 & 76.5 & 72.3 & 72.9 & 74.1 & 74.7 \\
Pichincha & 79.0 & 78.7 & 73.8 & 73.1 & 76.4 & 75.9 \\
Tungurahua & 77.7 & 76.9 & 72.5 & 72.2 & 75.1 & 74.5 \\
Zamora & 76.0 & 76.5 & 72.3 & 73.0 & 74.1 & 74.7 \\
Galápagos & 77.5 & 77.4 & 72.2 & 72.2 & 74.9 & 74.8 \\
Sucumbios & 76.0 & 75.9 & 72.3 & 71.9 & 74.1 & 73.9 \\
Orellana & 76.0 & 76.1 & 72.3 & 72.4 & 74.1 & 74.2 \\
Santo Domingo & 77.2 & 76.1 & 71.2 & 71.3 & 74.2 & 73.7 \\
Santa Elena & 78.1 & 77.5 & 74.8 & 73.2 & 76.5 & 75.3 \\
Nacional & 77.8 & 77.7 & 72.2 & 71.8 & 75.0 & 74.7 \\
\hline Fuente: elaboración del autor a partir de los datos del CPV 2010, Defunciones Generales 2010 y \\
2011 y Compendio Estadístico INEC 2015. & & & & \\
& & & & & &
\end{tabular}

Con el efecto de la interacción en los modelos encontramos que los habitantes del área rural siempre viven más que los del área urbana, pero el efecto del cambio de área en los hombres es mayor que el efecto del cambio de área en las mujeres con una diferencia de 0.29; por otro lado, la probabilidad de sobrevivir en las mujeres es siempre superior a la de los hombres aunque el efecto del cambio de sexo en el área urbana es mayor que el efecto del cambio de sexo en el área rural con una diferencia de 0.19. Adicionalmente, se encuentra que la probabilidad de sobrevivir de un habitante que es hombre y vive en el área rural es mayor que la de una mujer que vive en el área urbana. 
Considerando el método clásico actuarial encontramos que la esperanza de vida al nacer de los habitantes de Ecuador continental es 77.91 años, para las mujeres es 80.21 años y para los hombres es 75.62 años.

Con el método de Swanson, los valores de esperanza de vida al nacer son de 77.7, 71.8 y 74.7 años respectivamente para mujeres, hombres y todo Ecuador. Los resultados obtenidos con esta metodología resultan muy cercanos a los publicados por el INEC. Sin embargo, al no existir datos oficiales de esperanza de vida segregada por la variable área de residencia no se pudo ajustar un modelo a fin de estimar la esperanza de vida al nacer considerando el área urbana o rural y su interacción con el género.

En relación con el área de residencia, se obtuvo con el método clásico actuarial que una persona que vive en el área urbana tiene una esperanza de vida al nacer de 75.16 años y una persona que vive en el área rural 83.46 años. La interacción entre estas dos variables dio como resultado una esperanza de vida de 72.33 años para un habitante que es hombre y vive en el área urbana, 82.1 años para un habitante que es hombre y vive en el área rural, 77.98 para un habitante que es mujer y vive en el área urbana; $y$, 84.85 años para un habitante que es mujer y vive en el área rural.

Este último resultado contradice lo expuesto por Singh y Siahpush (2014) acerca de lo que acontece en Estados Unidos, esto podría ser originado entre otras causas por el problema de sub-registros que existen en datos de mortalidad en Ecuador, tal como lo indica el INEC (2012), debido a que en lo que se refiere a las defunciones existe una no correcta declaración de la edad para el periodo 1990-2010. Capa y Lara (2004) indican que el registro de defunciones del sector rural puede estar subestimado, probablemente porque las muertes de personas de este sector se podrían estar registrando en sitios urbanos, y con esto se obtenga una sobreestimación en el área urbana. Otro análisis hecho por la Comisión Especial de las Estadísticas de la Salud del Ecuador (2016) estima un sub-registro promedio de 33.13 por ciento para la mortalidad del Ecuador en 2011.

A fin de mejorar el registro tanto de las defunciones como de los nacimientos, se puede considerar lo que proponen Tomé et al. (1997), que esta actividad se centralice únicamente en el sector Salud (hospitales, centros de salud, clínicas o institutos de medicina legal), y que haya un sistema informático en línea que sea compartido por este sector y el Instituto Nacional de Estadística y Censos en el cual se alimente la información de muertes y nacimientos. Para que esto sea posible, es necesario que el personal que registre la información debe tener conciencia sobre la importancia de las estadísticas demográficas y ser capacitado para que haga el registro de 
nacimiento y defunción, a fin de disminuir el porcentaje de no registros. Otra cosa importante es crear conciencia en toda la población en general de la importancia del registro de nacimientos y muertes en el desarrollo económico del país, mediante la educación desde las escuelas, colegios, programas de televisión, etc., esto para reducir el no registro de nacimientos y muertes en las zonas rurales.

Si bien es cierto, en este estudio no se hizo una corrección de subregistros en los datos, lo que se refleja en los altos valores de esperanza de vida obtenidos por el método actuarial clásico, la metodología expuesta aquí resulta válida para el análisis de mortalidad en términos de determinar la existencia de otros factores, diferentes al sexo, que influyen en la misma, en este caso particular, el área de residencia.

\section{REFERENCIAS BIBLIOGRÁFICAS}

Acheampong, G. K. and Avorgbedor, Y. E., 2017, "Determinants of under Five Mortality in Ghana; A Logistic Regression Analysis Using Evidence from the Demographic and Health Survey (1988-2014)", in American Journal of Public Health Research, 5(3), 70-78.

Bureau, US Census, 2016, Health Insurance Coverage in the United States: 2016. Disponible en http://www.census.gov. Consultado el 2018-08-13.

Capa, H. and Lara, K., 2004, Construcción de tablas de mortalidad de la población ecuatoriana con base en el censo 2001 y estadísticas vitales. Memorias del IX Encuentro de Matemática y sus aplicaciones. Escuela Politécnica Nacional. Quito-Ecuador.

CEPAL, 2004, “América Latina: Tablas de Mortalidad 1950-2025”, en Boletín Demográfico. Santiago de Chile.

Chutinantakul, A., Mayeng, M. and Tongkumchum, P., 2014, "Estimation of mortality with missing data using logistic regression", in Songklanakarin J. Sci. Technol 36 (2), 249-254.

Comisión Especial de las Estadísticas de la Salud del Ecuador, 2016, Homologación del Cálculo de la Estimación del Sub-registro de mortalidad del Ecuador. Quito-Ecuador.

Davidian, M. and Giltinan, D., 1995, "Nonlinear models for repeated measurement data", in Monographs on Statistics and Applied Probability. Nueva York, Chapman and Hall/CRC. Disponible en http://www4.stat.ncsu.edu/ davidian/nlmmreview.pdf

Debón, A., Montes, F., and Sala, R., 2006, "A Comparison of Nonparametric Methods in the Graduation of Mortality: Application to Data from the Valencia Region (Spain)", in International Statistical Review / Revue Internationale De Statistique, 74(2), 215-233. Disponible en http://www.jstor.org/stable/25472704 
Guerrero, V. and Silva, E., 2015, "Smoothing a Time Series by Segments of the Data Range", in Communications in Statistics - Theory and Methods 44(1), 45684585, DOI: 10.1080/03610926.2014.901372

Guilkey, D. and Rindfuss R., 1987, "Logistic Regression Multivariate Life Tables: A Communicable Approach”, in Sociological Methods and Research 16(2): 276300 .

Gujarati, D. and Porter, D., 2010, Econometría, Mck-Graw Hill Interamericana. México

Instituto Nacional de Estadística y Censos, 2006, La mortalidad en el Ecuador: periodo 1990-2001. Quito-Ecuador, disponible en http://www.ecuadorencifras. gob.ec/documentos/webInec/Bibliotecas/Estudios/Estudios_Sociodemograficos/ La\%20Mortalidad\%20en\%20el\%20Ecuador\%20en\%20el\%20Periodo\%2019902001.pdf, (último acceso, 2/08/2017).

Instituto Nacional de Estadística y Censos, 2012, Proyecciones de la Población de la República del Ecuador 2010-2050. Quito-Ecuador. Tomado de http://www. ecuadorencifras.gob.ec/compendio-estadistico-2015/

Instituto Nacional de Estadística y Censos, 2015, Compendio Estadístico 2015. Quito-Ecuador.

Kleinbaum, D. and Klein, M., 2010, Logistic regression: a self-learning text. Statistics for Biology and Health. Springer. Nueva York- United Stated.

Navarro, C., González, E., Schmidt, J., Meneses, J. Martínez, J. and Ramírez, R., 2015, "Factores sociodemográficos y seguimiento prenatal asociados a la mortalidad perinatal en gestantes de Colombia", en Nutrición hospitalaria 32(3): 10911098. Madrid-España.

Newey, W. and McFadden, D., 1994, Large sample estimation and hypothesis testing. Handbook of econometrics. Elsevier Science. pp. 2111-2245.

Paredes, I. and Silva, E., 2017, "Estimación de la esperanza de vida a nivel municipal y por marginación sociodemográfica: una aplicación del método de Swanson para el caso de México, 2010”, en Estudios Demográficos y Urbanos 33(3). México.

Rivadeneira, L., 2008, Ecuador: Tablas Completas de Mortalidad. SENPLADES. Quito-Ecuador. Disponible en http://app.sni.gob.ec/sni-link/sni/Portal\%20 SNI\%202014/ESTADISTICA/Proyecciones_y_estudios_demograficos/07.pdf, (último acceso, 2/08/2017).

Sánchez, J., 2000, Construcción de una tabla de mortalidad para la población ecuatoriana. Escuela Superior Politécnica del Litoral. Guayaquil-Ecuador. Disponible en https:/www.dspace.espol.edu.ec/bitstream/123456789/4180/1/6708.pdf, (último acceso, 2/08/2017).

Silva, E., Guerrero, V. and Peña, D., 2014, "Suavizamiento controlado de tasas de mortalidad con P-splines: Aplicaciones para México y el Reino Unido", en Papeles de Población, 20(79), 99-131. 
Singh, G. and Siahpush, M., 2014, "Widening Rural-Urban disparities in Life Expectancy, U.S., 1969-2009”, in American Journal of Preventive Medicine 46(2): e19-e29. United Stated.

Swanson, David, 1989, "A state-based regression model for estimating substate life expectancy", in Demography, 26(1), 161-170.

Tomé, P., Reyes, H., Piña, C., Rodríguez, L. and Gutiérrez, G., 1997, “Características asociadas al subregistro de muerte en niños del estado de Guerrero, México", en Salud Pública de México 39(6), Fecha de consulta: 16 de agosto de 2018, Disponible en http://www.redalyc.org/articulo.oa?id=10639605, SSN 0036-3634

Vrieze, S. I., 2012, "Model selection and psychological theory: a discussion of the differences between the Akaike Information Criterion (AIC) and the Bayesian Information Criterion (BIC)", in Psychological Methods. United Stated, 17: 228-243, doi:10.1037/a0027127.

Wilson, J. and Lorenz, K., 2015, Modeling Binary Correlated Responses using SAS, SPSS and R. Springer. Nueva York, United Stated.

Yusuf, F., Martins, J. and Swanson, D., 2014, Methods of Demographic Analysis. Springer. Nueva York- United Stated.

Zhu, Z. and Zhi, L., 2014, "Logistic regression for insured mortality experience studies", in The Living to 100 Symposium. Orlando, Florida.

\section{RESUMEN CURRICULAR DE LOS AUTORES}

\section{Francisco Vera Alcívar}

Actualmente es Profesor de Estadística y Director del Departamento de Matemáticas de la Escuela Superior Politécnica del Litoral en Guayaquil, Ecuador. Tiene un doctorado en Estadística de la Universidad de Carolina del Sur en los Estados Unidos. Ha realizado investigaciones sobre procesos estocásticos y análisis de datos de vigilancia espacial-temporal. Actualmente investiga sobre el control estadístico de la calidad y los modelos lineales y no lineales.

Dirección electrónica: fvera@espol.edu.ec

\section{Mario Solórzano}

Master en Seguros y Riesgos Financieros, Analista de Proyectos de Investigación del Centro de Estudios e Investigación Estadística de la Escuela Politécnica del Litoral, durante el tiempo que ha trabajado en esta institución, ha participado en el desarrollo y ejecución de proyectos de investigación relacionados con la Análisis multivariado de datos y demografía. Dirección electrónica: mdsolorz@espol.edu.ec 


\section{Gina Ochoa}

Master en Seguros y Riesgos Financieros, Analista de Proyectos de Investigación en el Centro de Estudios e Investigación Estadística de la Escuela Politécnica Superior del Litoral, ha participado en la ejecución de varios proyectos de investigación en diferentes áreas estadísticas como el análisis de datos multivariados, muestreo y demografía. Además, ha desarrollado varios proyectos de investigación de mercado en particular en el área de la educación superior.

Dirección electrónica: vochoa@espol.edu.ec

\section{Sandra García Bustos}

Es profesora de estadística en la Escuela Superior Politécnica del Litoral de Guayaquil, Ecuador. En 2014, recibió su doctorado en Estadística y Optimización en la Universidad Politécnica de Valencia, España. Su investigación doctoral se centró en el estudio de los gráficos de control para las variables de Poisson correlacionadas. Tiene estudios de tercer nivel en Estadística y una Maestría en Administración de Empresas. Sus intereses actuales de investigación son el control estadístico de procesos y Estadísticas Sociales.

Dirección electrónica: slgarcia@espol.edu.ec

\section{Solange Cevallos}

Es profesora de estadística en la Escuela Superior Politécnica del Litoral de Guayaquil, Ecuador. Tiene estudios de tercer nivel en Estadística y una Maestría en Administración de Empresas. Actualmente está interesada en investigar diversas cuestiones relacionadas con las estadísticas con fines sociales.

Dirección electrónica: 1scevall@espol.edu.ec

Artículo recibido el 9 de agosto de 2017 y aprobado el 16 de julio de 2018. 